\section{Thromboseprophylaxe bei Knieendoprothetik: Aspirin gleichwertig zu Enoxaparin}

Radzak KN et al. Rate of Transfusions After Total Knee Arthroplasty in Patients Receiving Lovenox or High-Dose Aspirin. J Arthroplasty 2016; 31:

$2447-2451$

Bis heute besteht keine klare Evidenz, welches Medikament am besten als Thromboseprophylaxe in der Knieendoprothetik verwendet werden sollte. Radzak et al. haben in ihrer Studie die postoperativen Transfusions- sowie Thromboseraten nach implantierter Knieendoprothese unter der Gabe von Enoxaparin oder Aspirin verglichen.

In einigen Studien konnte bereits gezeigt werden, dass unter der Gabe von niedermolekularen Heparinen (NMH) gehäuft Komplikationen wie postoperative Hämatome oder vermehrt Bluttransfusionen auftraten. Daher präferieren einige Gesellschaften der Knieendoprothetik die postoperative Gabe von Aspirin, da hierunter eine ebenso effiziente Thromboseprophylaxe möglich sei.

\section{Methoden}

In der retrospektiven Studie wurden $377 \mathrm{~Pa}-$ tienten nach Implantation einer Knieendoprothese (288 uni-, 89 bilateral) nachuntersucht. Alle Patienten wurden von einem Operateur versorgt und nach festem Schema nachbehandelt. 179 Patienten erhielten Enoxaparin $40 \mathrm{mg}$ s.c. $1 \times$ täglich für $10 \mathrm{Ta}-$ ge und 198 Patienten Aspirin $325 \mathrm{mg} 2 \times$ täglich für 30 Tage postoperativ. Zur Beurteilung wurden der Hämoglobin- ( $\mathrm{Hb}$-) und Hämatokritwert (Hkt), die Tranfusionsrate sowie demografische Daten (Geschlecht, Alter, Gewicht, ASA-Klassifikation, BMI) erfasst.

\section{Ergebnisse}

Präoperativ bestanden ausgeglichene $\mathrm{Hb}$ Werte zwischen beiden Gruppen. Nach uni- und bilateraler Implantation einer Knieendoprothese war die Transfusionsrate in der Enoxaparin-Gruppe signifikant erhöht. Korrespondierend waren in dieser Gruppe der Hb- und Hkt-Abfall postoperativ vermehrt und die Hospitalisationszeit verlängert. Bezüglich der Inzidenz einer tiefen Venenthrombose (TVT) oder Lungenembolie (LE) gab es keine signifikanten Unterschiede (Aspirin: 4 TVT, 3 LE; Enoxaparin 2 TVT, 3 LE).

\section{FAZIT}

Die Studie zeigt, dass nach Implantation einer Knieendoprothese die Gabe von hochdosiertem Aspirin postoperativ als Thromboseprophylaxe ebenso effektiv ist wie die Gabe von NMH bei signifikant verringerten postoperativen Blutungskomplikationen. Kritisch anzumerken ist die fehlende Randomisierung der beiden Gruppen. Eine prospektiv-randomisierte Studie sollte durchgeführt werden, um die Effizienz von NMH mit Aspirin als Thromboseprophylaxe nach Implantation einer Knieendoprothese zu vergleichen.

Dr. Sarah Ettinger, Hannover 